\title{
A Bose-Einstein condensate in a random potential
}

\author{
J. E. Lye*, L. Fallani, M. Modugno ${ }^{1}$, D. Wiersma, C. Fort, and M. Inguscio \\ LENS, Dipartimento di Fisica, and INFM Università di Firenze via Nello Carrara 1, I-50019 Sesto Fiorentino (FI), Italy \\ 1 also BEC-INFM Center, Università di Trento, I-38050 Povo (TN), Italy
}

\begin{abstract}
An optical speckle potential is used to investigate the static and dynamic properties of a BoseEinstein condensate in the presence of disorder. For strong disorder the condensate is localized in the deep wells of the potential. With smaller levels of disorder, stripes are observed in the expanded density profile and strong damping of dipole and quadrupole oscillations is seen. Uncorrelated frequency shifts of the two modes are measured for a weak disorder and are explained using a sum-rules approach and by the numerical solution of the Gross-Pitaevskii equation.
\end{abstract}

PACS numbers: 03.75.Kk, 03.75.Lm, 42.25.Dd, 32.80.Pj

The nature of a bosonic system in the presence of disorder has been intensely explored both theoretically and experimentally, particularly in the context of ${ }^{4} \mathrm{He}$ in a porous material, where the breakdown of superfluidity with sufficient disorder was observed [1]. In the zero temperature limit, theoretical studies have provided the striking result that there exists a regime where the superfluid fraction is significantly smaller than the condensate fraction [2]. A further consequence of disorder is the quantum phase transition from a superfluid to a localized Bose-Glass state [3]. Indications of such a state have been found in several physical systems but the precise phase diagram of the progression from superfluid, to Bose-Glass, to Mott-insulator state remains unclear [4]. Furthermore, Anderson localization occurs for weakly interacting bosons [5], and plays an important role in solid state physics where electron transport can be disrupted by defects in a solid [6], and more recently was observed for photons in strongly scattering semiconductor powders 7].

The realization of Bose-Einstein condensation (BEC) in a dilute alkali vapor offers the possibility of an ideal system in which to explore the rich arena of disorder related phenomena. Already there has been considerable work on the superfluidity and long-range coherence properties of these degenerate Bose gases, and with the combination of BEC and optical lattices there has been an explosion of observations on phenomena of solid statephysics [8], notably including the superfluid to MottInsulator quantum phase transition [9]. Recently, theoretical works have discussed the possibility of Bose-Glass and Anderson localization transition in a BEC in a disordered optical lattice [10, 11]. Understanding the effect of disorder is also important for BEC in microtraps where fragmentation of the trapped BEC density profile has been observed and is now attributed to intrinsic disorder in the fabrication of the microchip 12, 13]. It is crucial for integrated atom optics on microchips to quantitatively define how this disorder will change the coherence and transport properties, particularly considering that in 1D disordered systems localization of the excitations could play an important role [14].

We present in this Letter the first results on a Rb BEC in a disordered potential, where the precise realization of
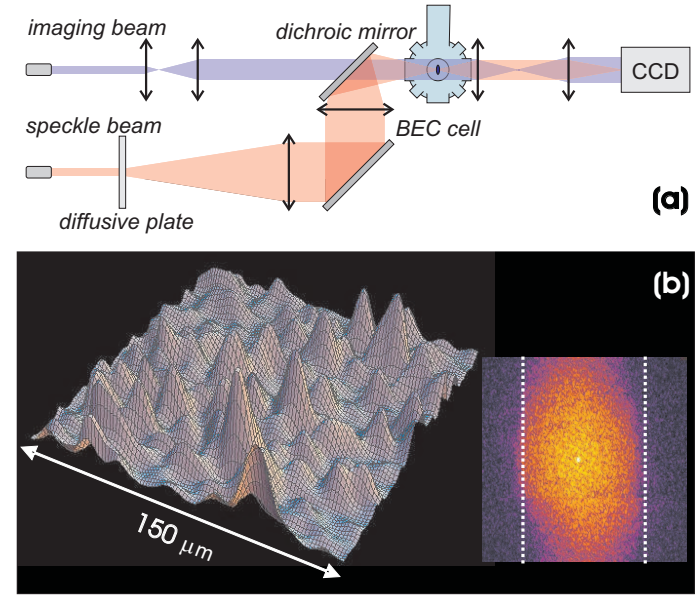

FIG. 1: (a) Optical setup for both the speckle potential and the imaging beam for the BEC. The axial direction of the magnetic trap is in the vertical direction of the figure. (b) $3 \mathrm{D}$ representation of the speckle potential (left) and its Fourier transform (right). The dotted lines correspond to a length scale of about $10 \mu \mathrm{m}$ in the axial direction.

the disorder is controllable. We consider both the limit of strong disorder, where we observe that the BEC is localized in the wells of the high random potential, and the situation where the disorder is smaller than the chemical potential of the BEC, where we see stripes in the expanded density profile and damping of dipole oscillations. In the perturbative limit of very weak disorder we observe shifts in the frequency of the quadrupole excitation mode.

To produce the random potential a laser beam is shone through a diffusive plate. The beam is derived from a Ti:Sa laser at $\lambda=822 \mathrm{~nm}$, far detuned with respect to the $\mathrm{Rb}$ D1 line at $\lambda=795 \mathrm{~nm}$. The resulting speckle pattern is imaged onto the trapped BEC, as shown in Fig. 1(a), producing a two-dimensional (2D) potential perpendicular to the beam propagation, that varies spatially but is stable in time. A nice feature of the optical setup is that the path of the speckle beam is combined with the path of the imaging beam using a dichroic mir- 
ror $(\lambda>800 \mathrm{~nm}$ reflected, $\lambda<800 \mathrm{~nm}$ transmitted). The imaging setup is used to detect both the BEC and the speckle pattern, and thus we can image in consecutive photos the position of the trapped BEC and the precise realization of the random potential that the $\mathrm{BEC}$ experiences. The BEC is produced in a Ioffe-Pritchard magnetic trap, elongated perpendicular with respect to the speckle beam. The trapping frequencies are $\omega_{z}=$ $2 \pi \times(8.74 \pm 0.03) \mathrm{Hz}$ axially and $\omega_{\perp}=2 \pi \times(85 \pm 1) \mathrm{Hz}$ radially, with the axis of the trap oriented horizontally. Our typical BECs are made of $\simeq 3 \times 10^{5}$ atoms in the hyperfine ground state $\mid F=1 ; m_{F}=-1>$, with a peak density $n \simeq 1.2 \times 10^{14} \mathrm{~cm}^{-3}$.

A $3 \mathrm{D}$ representation of a typical speckle potential is displayed in Fig. I(b). In the right part Fig. [1 we show the Fourier transform of this potential, where the smallest length scale of the speckle is $10 \mu \mathrm{m}$. The average distance between neighboring speckles is approximately $20 \mu \mathrm{m}$. The axial and radial size of our BEC is $110 \mu \mathrm{m}$ and $11 \mu \mathrm{m}$ respectively. The BEC probes 6 wells in the axial direction and only 1 well on average in the radial direction, and thus the cylindrical geometry enforces a quasi-one dimensionality on the speckle-condensate system. We define the speckle height $V_{s}$ by taking twice the standard deviation of the speckle potential over a distance of $200 \mu \mathrm{m}$ along the BEC axial direction. The speckle height is expressed throughout this Letter in units of frequency.

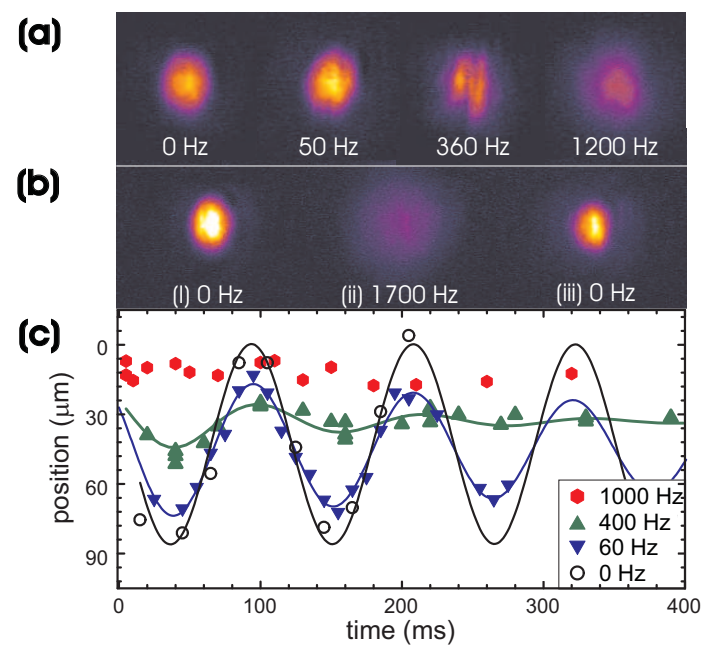

FIG. 2: (a) Density profile of the BEC after $28 \mathrm{~ms}$ of expansion from the combined magnetic and speckle potential for varying speckle potential intensities $V_{s}$ (indicated in bottom part). To verify that the transfer to the speckle is adiabatic (b) shows the density profiles with (i) no speckle potential, (ii) when the speckle potential is abruptly switched off, and (iii) when it is adiabatically ramped off. (c) Dipole oscillations in the combined magnetic and speckle potential for varying $V_{s}$.

An initial insight into the effect of the random potential can be obtained by considering the BEC in expansion. Fig. 2(a) shows a series of absorption images af- ter $28 \mathrm{~ms}$ of free expansion for different values of $V_{s}$. In each image the speckle intensity is increased adiabatically using a $200 \mathrm{~ms}$ exponential ramp with a time constant of $40 \mathrm{~ms}$. After $50 \mathrm{~ms}$ in the combined magnetic and speckle potential, both potentials are abruptly switched off. For $V_{s} \sim 50 \mathrm{~Hz}$ much lower than the chemical potential of the BEC in the harmonic trap $(\sim 1 \mathrm{kHz})$, no change from the standard Thomas-Fermi (TF) profile is seen. As the potential is increased to an intermediate power $(\sim 300 \mathrm{~Hz})$ we see a strong modification in the expanded density profile. Such modifications can be a signature of the development of different phase domains across the BEC, similar to the stripes seen with highly elongated quasi-condensates [15, 16], or when different momentum components are present due to the growth of instabilities [17]. We note that the spacing of the stripes we observe, $>50 \mu \mathrm{m}$, can not be related to the characteristic length scale of the speckle pattern, $10-20 \mu \mathrm{m}$, the latter would give rise to an interferogram length scale of 6-12 $\mu \mathrm{m}$ after $28 \mathrm{~ms}$ of expansion. Finally, when $V_{s}>1 \mathrm{kHz}$, a value greater than the chemical potential, the system enters the tight-binding regime. Here in expansion only a broad gaussian profile is seen. The tunnelling between wells is expected to be completely suppressed for such large spacing of adjacent sites $(>10 \mu \mathrm{m})$, and we are seeing the expansion of an array of randomly spaced sources. Unlike the situation of the expansion of a 1D array of equally spaced sources with random phases where a periodic interference pattern is still visible in a single run [18], no regular interference is expected to be seen with random spacing either for uniform or for random phases. To check that the ramp is adiabatic, we have ramped the speckle on (to our maximum speckle height of $1.7 \mathrm{kHz}$ ) and then off, using the exponential ramp described earlier. Fig. 2(b)(i) shows the expanded BEC density profile with no speckle, (ii) the expanded profile when the speckle potential is abruptly switched off, and (iii) the recovered TF profile when the speckle intensity is adiabatically decreased. This also clearly indicates that the density profile in Fig. 2(b)(ii) can not be attributed to heating of the atomic cloud.

The transport properties in each of the above regimes are investigated by abruptly shifting the magnetic trap in the axial direction by $25 \mu \mathrm{m}$ inducing dipole oscillations shown in Fig. 2(c). Undamped oscillations are observed in the absence of the speckle. The dipole motion for very weak $V_{s}(\sim 60 \mathrm{~Hz})$ is slightly damped with an unchanged frequency, and in the intermediate $400 \mathrm{~Hz}$ potential, where phase fragmentation is seen in expansion, strong damping is observed. Once in the tight-binding regime, $V_{s}>1 \mathrm{kHz}$, the atomic cloud does not oscillate and remains localized in the deep, largely spaced, wells of the speckle potential on the side of the magnetic trap. This is analogous to the 'pinning' effect seen in magnetic microtraps, where the axial frequency is relaxed but the atoms do not propagate along the newly created waveguide remaining localized in the local minima due to the disorder in the microchip [13. 

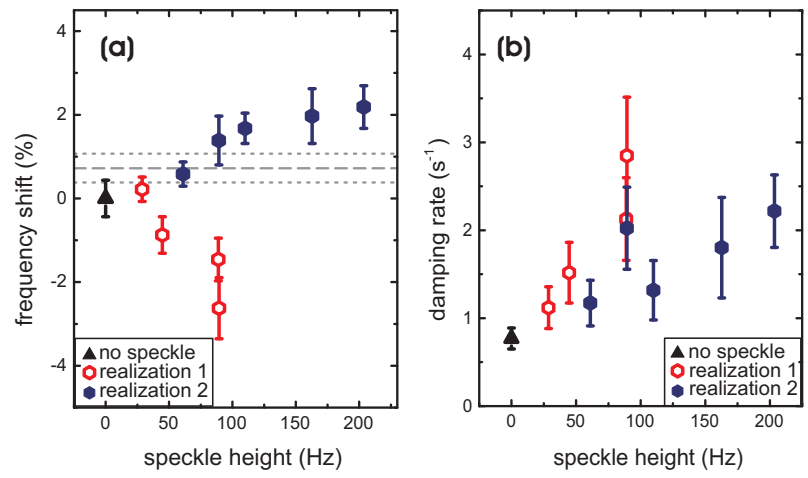

FIG. 3: Quadrupole oscillations with increasing $V_{s}$ for two different speckle realizations: (a) frequency shift (relative to the quadrupole frequency $13.72 \pm 0.06 \mathrm{~Hz}$ measured in the pure harmonic trap), and (b) damping rate. The dashed line corresponds to $\sqrt{5 / 2} \nu_{d}$, calculated from the measured dipole frequency of $8.74 \pm 0.03 \mathrm{~Hz}$. The error (dotted lines) reflects the variation of the trap over several months.

Precise information on the nature of BEC has been gained through studies of collective excitations [19] and it is therefore a natural starting point to gather information on a BEC in a random potential. We measure the frequency of both the dipole, $\nu_{d}$, and quadrupole, $\nu_{q}$, modes of a BEC confined in the harmonic magnetic trap combined with a weak speckle potential in the range of 30 - $200 \mathrm{~Hz}$ corresponding to a small perturbation of the harmonic potential. The quadrupole mode is excited with a resonant modulation of the radial trapping frequency at $13.7 \mathrm{~Hz}$, producing oscillations of $5 \%$ of the trapped BEC axial width, while the dipole mode is excited by an abrupt shift of the trap of $25 \mu \mathrm{m}$ in the axial direction. In both cases the speckle potential remains stationary. The results for the frequency shift of the quadrupole oscillations versus $V_{s}$ for two different speckle realizations are shown in Fig. [3 with the corresponding damping of this mode. The shift is taken relative to the measured quadrupole frequency, $13.72 \pm 0.06 \mathrm{~Hz}$, in the pure harmonic trap. We see frequency shifts of up to $3 \%$, and also observe that both the sign and amplitude of the shift depend on the exact realization of the speckle potential. We measure no change in the dipole frequency within our experimental resolution of $1 \%$. These measurements are in contrast to what is seen with a regular standingwave optical lattice, where both $\nu_{d}$ and $\nu_{q}$ are rescaled with the single particle effective mass due to the optical lattice 20].

In the absence of the speckle potential, the quadrupole mode of an elongated BEC is given by $\nu_{a}=\sqrt{5 / 2} \nu_{d}$ in the zero-temperature TF approximation [21]. In the presence of an additional potential, this value can be modified by both the deviation of the potential from a pure harmonic oscillator, and other effects such as changes in the condensate and superfluid fraction, modification of the interatomic interactions, or an increase in the ther-
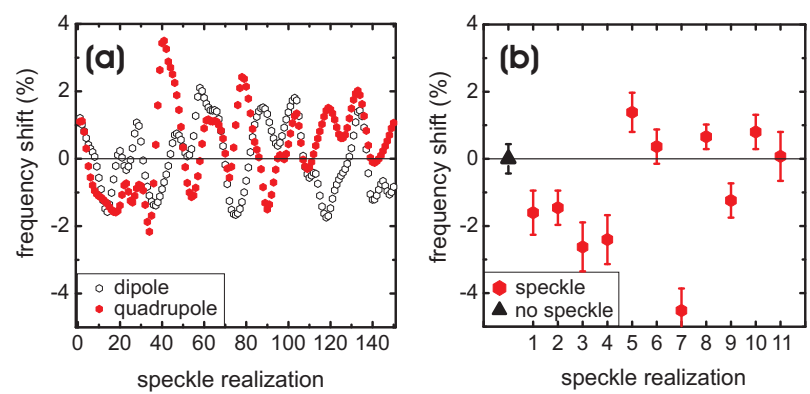

FIG. 4: (a) Dipole and quadrupole frequency shifts for 150 slices of the same speckle realization calculated from the sum rules approach. (b) Measured shift in the quadrupole frequency for 11 different realizations of the speckle at a fixed height of $80 \pm 10 \mathrm{~Hz}$.

mal component. For example, in the collisional hydrodynamic limit, calculated for a gas above the condensation temperature, the frequency of the quadrupole mode in a harmonic potential is $\nu_{q}=\sqrt{12 / 5} \nu_{d}$, while in the noninteracting thermal limit $\nu_{q}=2 \nu_{d}$ [22]. Actually, even without the speckle potential, our measured quadrupole frequency is shifted $-0.7 \%$ with respect to the predicted $\sqrt{5 / 2} \nu_{d}$, similar to what has been measured previously and attributed to the presence of a residual thermal component 23]. To check that the observed frequency shifts with the speckle present are not simply an effect of heating, we have measured the quadrupole frequency in the presence of a large thermal fraction (50\%) observing only a small shift of $-0.4 \%$ with respect to the measurement with no discernable thermal fraction.

To understand the effect of a shallow random potential on the dynamics of the BEC we use two theoretical approaches. The Gross-Pitaevskii equation (GPE) is solved in the actual potential used in the experiment, and the numerical predictions are confirmed using a sum-rules approach. We investigate the dynamics of the BEC with the GPE in the combined speckle, $V_{o p t}$, and harmonic, $V_{h o}$, potential

$$
i \hbar \frac{\partial \Psi}{\partial t}=\left(-\frac{\hbar^{2}}{2 m} \nabla^{2}+V_{o p t}+V_{h o}+\frac{4 \pi \hbar^{2} a}{m}|\Psi|^{2}\right) \Psi(1)
$$

where $\Psi$ is the complex BEC order parameter, $m$ is the atomic mass, and $a$ is the scattering length. The GPE is solved after a sudden displacement $\Delta z$ of the harmonic trap for the dipole mode or after a sudden change in the axial trapping frequency for the quadrupole mode, for a variety of different speckle realizations. In the case of small amplitude oscillations $(\Delta z=3 \mu \mathrm{m}$ and quadrupole oscillations of $5 \%$ of the BEC aspect ratio), the BEC oscillates coherently with no appreciable damping for simulations up to $600 \mathrm{~ms}$. The frequencies are red or blue shifted depending on the particular speckle realization, and in some configurations a red shift is observed for one mode and a blue shift for the other. The result for the quadrupole mode shows an increasing frequency shift 
with increasing $V_{s}$ as qualitatively confirmed by the experimental results in Fig. B 3 . We found that the predicted frequency shift of the dipole mode reduces for larger amplitude oscillations since in this case the BEC probes more of the outer unperturbed harmonic oscillator potential, and less of the central perturbed potential. Significant frequency shifts are observed $(>1 \%)$ only for small amplitude oscillations $(\Delta z<10 \mu \mathrm{m})$, but unfortunately we do not have sufficient experimental accuracy with the small amplitude oscillations to measure these shifts. The somewhat surprising behavior predicted by the GPE of disparate dipole and quadrupole frequency shifts can be explained by using the sum rules approach and considering $V_{\text {opt }}$ as a perturbation. According to the sum rules, the frequencies of the low lying collective excitations can be estimated as $\hbar^{2} \omega^{2}=m_{3} / m_{1}$ where the moments $m_{i}$ can be expressed as commutators involving the many-body hamiltonian $H$ and a suitable excitation operator $F[19]$. In our case this operator can be chosen as $F_{d} \sim z$ and $F_{q} \sim r^{2}-\alpha z^{2}$ for the dipole and quadrupole modes respectively, $\alpha$ being a variational parameter 24]. Treating the speckle potential $V_{o p t}$ as a small perturbation, and writing $\omega^{2}=\omega_{0}^{2}+\Delta$ we get

$$
\begin{aligned}
\Delta_{d} & \simeq \frac{1}{m}\left\langle\partial_{z}^{2} V_{o p t}\right\rangle_{0} \\
\Delta_{q} & \simeq \frac{1}{m} \frac{\left\langle z \partial_{z} V_{o p t}+z^{2} \partial_{z}^{2} V_{o p t}\right\rangle_{0}}{\left\langle z^{2}\right\rangle_{0}}
\end{aligned}
$$

where the second line is obtained assuming a strongly elongated BEC. These analytical results demonstrate how the dipole and quadrupole frequencies have different dependencies on the particular characteristics of the perturbing potential. We have verified that these predictions are in agreement with the full solution of the GPE in the cases considered.

In Fig. 4(a) we show the predictions of the sum rules for 150 realizations of the speckle potential, with $V_{s} \sim$ $30 \mathrm{~Hz}$. Each realization is obtained by taking successive $1 \mathrm{D}$ slices of $1 \mu \mathrm{m}$ from the same $2 \mathrm{D}$ experimental speckle pattern. The average shift is close to zero for both excitation modes. We have performed a similar series of measurements experimentally. Fig. 4(b) shows the quadrupole frequency for different realizations of the speckle potential at $V_{s} \sim 80 \mathrm{~Hz}$. As predicted, we observe both red and blue shifts, however within our limited statistics we see a bias towards the red. We have also begun an exploration of the dipole and quadrupole modes for larger amplitude oscillations where the solution of the GPE indicates that the superfluidity of the system may be compromised. The simulations show short wavelength modulation of the density distribution and damped oscillations. Also in the experiment we found first evidence of increased quadrupole frequency shifts and strong perturbations of the expanded density profile in this regime.

In summary, we present the first results on a BEC in a controllable random speckle potential. We see the effect of a weak random potential indicated by stripes in the expanded density profile of the BEC, damped dipole oscillations, and frequency shifts in the quadrupole mode that are not correlated to the measured dipole frequency. Simulations solving the GPE and an analytical sum-rules approach successfully describe these shifts in the pertubative regime. In the limit of a strong random potential, we observe a broad gaussian density profile after expansion, indicating that the atoms remain localized in the individual wells of the speckle potential. This opens the way to new investigations of localization phenomena in quantum gases, and the superfluid behavior with varying roughness of a surface potential.

This work has been supported by the EU Contracts No. HPRN-CT-2000-00125, INFM PRA "Photon Matter" and MIUR FIRB 2001. J. E. L. was supported by EU. We acknowledge for stimulating discussions G. Shlyapnikov, R. Roth, J. Zakrzewski, S. Stringari and colleagues from the INFM-BEC Center in Trento, and all the people of the Quantum Gases group in Florence. We also thank F. S. Cataliotti, V. Guarrera and M. Scheid for experimental assistance and discussions.
[*] Electronic address: lye@lens.unifi.it

[1] J. D. Reppy, J. Low Temp. Phys. 87, 205 (1992) and references therein.

[2] G. E. Astrakharchik, J. Boronat, J. Casulleras, and S. Giorgini, Phys. Rev. A 66, 023603 (2002); S. Giorgini, L. Pitaevskii, S. Stringari, Phys. Rev. B 49, 12938 (1994); K. Huang and H. F. Meng, Phys. Rev. Lett. 69, 644 (1992); A. V. Lopatin and V. M. Vinokur, Phys. Rev. Lett. 88, 235503 (2002).

[3] M. P. A. Fisher, P. B. Weichman, G. Grinstein, D. S. Fisher, Phys. Rev. B 40, 546 (1989); R. T. Scalettar, G. G. Batrouni, and G. T. Zimanyi, Phys. Rev. Lett. 66, 3144 (1991); W. Krauth, N. Trivedi, and D. Ceperley, Phys. Rev. Lett. 67, 2307 (1991).

[4] N. Markovic, C. Christiansen, A. M. Mack, W. H. Huber, and A. M. Goldman, Phys. Rev. B 60, 4320 (1999);
P. A. Crowell, F. W. Van Keuls, and J. D. Reppy, Phys. Rev. B 55, 12620 (1997); H. S. J. van der Zant, W. J. Elion, L. J. Geerligs, and J. E. Mooij, Phys. Rev. B 54, 10081 (1996).

[5] P. W. Anderson, Phys. Rev. 109, 1492 (1958).

[6] See, e.g., P. A. Lee and T. V. Ramakrishnan, Rev. Mod. Phys. 57, 287 (1985); Ping Sheng, Introduction to Wave Scattering, Localization, and Mesoscopic Phenomena (Academic Press, New York, 1995).

[7] R. Dalichaouch et al., Nature 354, 53 (1991); D. S. Wiersma et al., Nature 390, 671 (1997).

[8] J. H. Denschlag et al., Journ. of Phys. B 35, 3095 (2002).

[9] M. Greiner et al., Nature 415, 39 (2002).

[10] R. Roth and K. Burnett, Phys. Rev. A 68, 023604 (2003).

[11] B. Damski, J. Zakrzewski, L. Santos, P. Zoller, and M. Lewenstein, Phys. Rev. Lett. 91, 080403 (2003). 
[12] S. Kraft et al., J. Phys. B 35, L469 (2002); A. E. Leanhardt et al., Phys. Rev. Lett. 90, 100404 (2003); J. Estève et al., Phys. Rev. A 70, 043629 (2004); D. W. Wang, M. D. Lukin, and E. Demler, Phys. Rev. Lett. 92, 076802 (2004).

[13] J. Fortágh, H. Ott, S. Kraft, A. Günther, and C. Zimmermann, Phys. Rev. A 66, 041604(R) (2002).

[14] G. Shlyapnikov, private communication.

[15] S. Dettmer et al., Phys. Rev. Lett. 87, 160406 (2001).

[16] S. Richard et al., Phys. Rev. Lett. 91, 010405 (2003)

[17] L. Fallani et al., Phys. Rev. Lett. 93, 140406 (2004).

[18] Z. Hadzibabic, S. Stock, B. Battelier, V. Bretin, and
J. Dalibard, Phys. Rev. Lett. 93, 180403 (2004).

[19] F. Dalfovo et al., Rev. Mod. Phys. 71, 463 (1999).

[20] C. Fort et al., Phys. Rev. Lett. 90, 140405 (2003).

[21] S. Stringari, Phys. Rev. Lett. 77, 2360 (1996).

[22] A. Griffin, Wen-Chin Wu, S. Stringari, Phys. Rev. Lett. 78, 1838 (1997).

[23] D. M. Stamper-Kurn, H.-J. Miesner, S. Inouye, M. R. Andrews, and W. Ketterle, Phys. Rev. Lett. 81, 500 (1998).

[24] T. Kimura, H. Saito, and M. Ueda, J. Phys. Soc. Jpn., 68, 1477 (1999). 